\title{
Research on Fault-feeder Selection Dvice
}

\author{
Ziming Zhuo ${ }^{1}$, Haimeng $\mathrm{Sun}^{2}$, Haibin $\mathrm{Ji}^{1}$ \\ ${ }^{1}$ School of Mechanical \& Electrical Engineering Xuzhou College of Industrial Technlolgy, Xuzhou, China \\ ${ }^{2}$ Xuzhou Distribution Station Xuzhou Power Supple Company, Xuzhou, China \\ Email: zimingzhuo@126.com
}

Received February, 2013

\begin{abstract}
The compensation current of the arc-suppressing coil makes the phase and amplitude of zero-sequence measurement current of the earthed fault feeder to vary. It is very hard to detect the fault feeder by using existing detectors based on single method. In this paper, integrative feeder selection strategy-zero sequence current increment method and the direction of transient current - is put forward. Based on the integrative feeder selection strategy, the design of fault-feeder selection device for one-phase-to ground fault on resonance grounding system is presented. For the purpose of testing and validating the operating principle of the device, the experiment of single-phase-to-ground fault has been carried out on the simulation of $1.2 \mathrm{kV}$ power network. The results from many repeat experiments show that stability of the fault selection device is satisfactory.
\end{abstract}

Keywords: Resonance Grounding System; Earthed Fault Feeder Detection; Zero-Sequence Current Increment Method; Zero-sequence Transient Currents

\section{Introduction}

Neutral ineffectively grounding modes especially resonance grounding systems are widely employed in medium and low voltage distribution networks of China. Because of the compensation current of the arc-suppressi ng coil, the fault current is so weak that it is hard to detect fault-feeder. When the single-phase-to-ground fault occurs in resonance grounding system, it is an important research subject in resonance grounding system that detecting the ground fault line.[1,2]

Ground-fault is complicated in resonance grounding system. Zero sequence current may only contain transient or steady-state or both of them in all kinds of groundault. The existing detectors are based on transient analysis or steady analysis of zero-sequence current. It makes the result of detecting is not satisfactory.

This paper presents a new method of integrative faultfeeder selection that is based on the steady-state analysis current zero-sequence current increment and the direction of transient current analysis zero sequence transient current direction.

\section{Method of Integrative Fault-feeder Selection}

\subsection{Zero Sequence Current Increment Method}

When single phase grounding fault occurs in resonance grounding system, zero-sequence transient current of non-fault line.

$$
I_{0}=3 U_{0} / R_{1}+j 3 U_{0} \omega C_{1}
$$

where $R_{1}$ represents each line's the resistance to ground, $C_{1}$ represents each line's capacitance to earth.

When single-phase-to-ground fault takes place, the compensation current of arc-suppressing coil makes phase and amplitude of the zero-sequence measurement current of earthed fault feeder to vary. $\Delta I_{0 i}$ represents decrease of the zero-sequence current of non-fault feeder with and without compensation .

$$
\Delta I_{0 i}=I_{0 i 2}-I_{0 i 1}=\left(U_{02}-U_{01}\right)\left(\frac{3}{\mathrm{R}}+\mathrm{j} 3 \omega_{\mathrm{i}}\right)
$$

$L_{1}$ represents inductance value of arc-suppressing before adjustment, $L_{2}$ represents inductance value of arcuppressing after adjustment, then $\Delta I_{0 \mathrm{k} 1}$ represents zero sequence current of fault feeder. $\Delta I_{0 \mathrm{k}}$ epresents zero sequence current difference values of fault-feeder before and after adjusting inductance value[4,5,6].

$$
I_{0 \mathrm{k} 1}=-U_{01}\left(\sum_{i=1, i \neq \mathrm{k}}^{n} \frac{3}{\mathrm{R}_{i}}+\sum_{i=1, i \neq \mathrm{k}}^{n} j 3 \omega c_{\mathrm{i}}+\frac{1}{\mathrm{R}_{n}}-j \frac{1}{\omega L_{1}}\right)
$$

$\Delta I_{0 \mathrm{k}}$ not only relates to parameters $U_{01}, U_{02}$, but also depends on parameters $L_{1}$.

The value of $U_{01}-U_{02}$ changed slightly, even entirely neglect the influence on the result of $\Delta I_{0 \mathrm{k}} \cdot \Delta I_{0 \mathrm{k}}$ is affected remarkably by $L_{1}$. Through the discussion of the 
Equation (3), (4), it is found that Effective value of $\Delta I_{0 \mathrm{k}}$ is higher than those of $\Delta I_{0 \mathrm{k}}$, which provides operational judgment basis for fault feeder selection.

$$
\left.\Delta I_{0 \mathrm{k}}=\left(U_{01}-U_{02}\right)\left(\sum_{i=1, i \neq \mathrm{k}}^{n} \frac{3}{\mathrm{R}}+\sum_{i=1, i \neq \mathrm{k}}^{n} j 3 \omega c_{\mathrm{i}}+\frac{1}{\mathrm{R}_{n}}\right)+j \frac{U_{02} \mathrm{~L}_{1}-U_{01} \mathrm{~L}_{2}}{\omega L_{1} L_{2}}\right)(4)
$$

\subsection{The Transient Current Direction Method}

When single-phase earth fault occurs in resonance earthed distribution system, for some cases the steady state component of fault current is small or approximates to zero, it leads to insufficient faulty feeder detection that is based on steady state analysis. According to the sign of integral mean value of mutual dot products of zero-sequence transient currents of each two feeders, an approach to judge the direction of fault current is proposed. The integral mean value during a time interval are taken as the judging quantity

If the ith line and jth line are non-fault line, the fault judging quantity of every feede was the product of two lines zero-sequence transient current $[1,3,4]$

$$
i_{i}(t) \cdot i_{j}(t)=i_{0 f \cdot i}(t) \cdot i_{0 f \cdot j}(t) \geq 0
$$

If one of the ith line and jth line is fault line, $P_{i}$ was the product of two lines of zero-Sequence transient current

$$
i_{i}(t) \cdot i_{j}(t)=i_{0 f \cdot i}(t) \cdot i_{0 g \cdot k}(t) \leq 0
$$

The following conclusions are drawn from above analysis: Only one $P_{i}$ is negative integer and the rest is positive integer. ith feeder is fault feeder. All of $P_{i}$ are positive integer and Reference feeder is fault feeder

All of $P_{i}$ are positive integer and Reference feeder is fault feeder.

\section{Software and Hardware Composition of Fault-line selection Device}

\subsection{Hardware Composition}

Fault-line selection device for One-phase-to-ground Fault on resonance grounding system is designed from both hardware and software. Hardware properties directly affect the performance of device. According to the design requests, the detecting equipment use chip TMS320LF2812A of TI Company as central processor. Zero sequence voltage analog signals and zero sequence current analog signals of feeders, which is collected through the signal acquisition module, will be converts into digital signal, and real-time display of calculated results of zero sequence current value. Based on integrative line selection strategy-zero sequence current increment method and the direction of transient current, the device judge whether the occurring of the single phase-to-earth fault. Hardware comprises main components-power supply, A/D converter, central processor, signal sampling circuit.

\subsection{Software Composition}

Major task for software system of fault-feeder selection is real-time sampling of zero sequence voltage and zero sequence current. The data can be used to further analysis and judgment of the operating state of electric network.

The software design adopts the module design thought, and takes the master routine and the interrupt subroutine as the main structure. The program consists of one main program, and four subroutines (system initialization module, Signal sampling module, Fault judgment module, HMI module).

First, system is initialized, then the main loop Proceed to start sampling. As it can be seen from Figure 1: When single-phase grounding fault occurs, the system calls fault judgment subroutines, records the fault information and the fault-line detection result is displayed on the LCD screen. Under normal operation condition of the network, the main program has been in the acquisition and processing of zero sequence current and zero sequence voltage. When there is an interrupt request signal, DSP deals with corresponding subroutines.

The sampling program adopts time interruption acquisition. a common timer of The EVA (event management a timer ) is used as sampling timer .For each sampling interval, EVA generate a timing interrupt. Based on the timing interrupt, AD converter puts 10-channel analog signals of zero sequence voltage and current into Digital Signal. When is called again, AD converter puts module put analog signals of zero sequence voltage and current into digital signal by Multiplex[7].

When it is detected that zero-sequence voltage is larger than setting value, block diagram of fractional subroutines is called subroutine and examine the fault-line by analysis and judgment of Sampling zero-sequence voltage and zero-sequence current program flow chart is given as follows:

For the method of the transient current fault-feeder selection, notch filter for power-line interference is applied to sampling data to obtain amplitude of zero sequence current. Maximum amplitude of zero sequence current can be considered to be reference feeder. The fault judging quantity of every feeder $P_{i}$ is the product of the sampling zero sequence current values of reference feeder and other feeders. Fault-feeder is then identified according to formula 4. Every fault-feeder is given a set of only numbers after the judgment of fault-feeder, so sampling data array includes a row of numbering of each line. 


\section{Experiment of Selection Device}

Once the device of fault line selection has been designed and constructed, its experimental performance has been tested.

\subsection{Zero-sequence Measurement Current of Non-faulted Fault-feeder}

For the purpose of validating the selection, the experiment of one-phase-to-ground fault on resonance grounding system has been carried through with the simulation of $1.2 \mathrm{kV}$ power network. As it has been done in the preceding experimentation, real wareform of fault and nonfault zero sequence current is obtained by fault wareform recording.

As it can be seen from Figure 2: From the top to the end, it is waveform of 1 st and 2nd zero-sequence measurement current of non-fault feeder, 3rd zero-sequence measurement current of non-fault feeder, zero sequence voltage. MHK series controller of arc suppression coil start to adjust the inductance of arc suppression coil after 5 cycles.

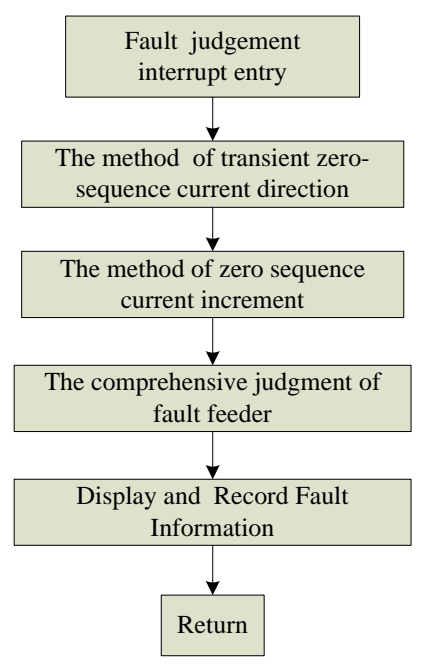

Figure 1. Block diagram of fractional subroutines.

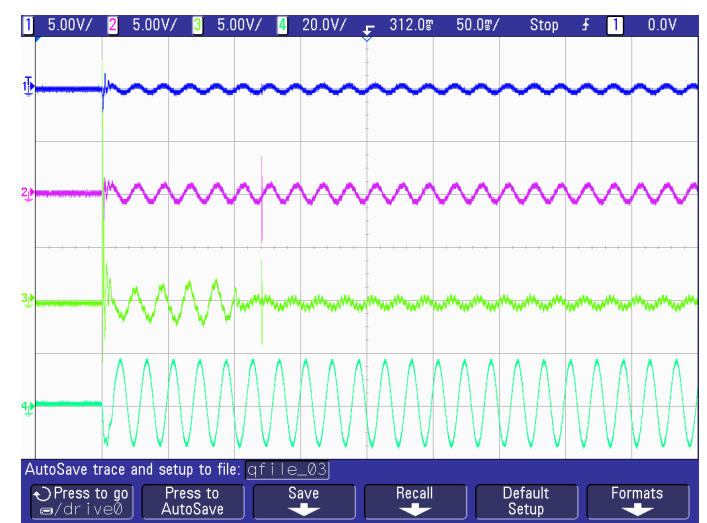

Figure 2. wave recording of one-phase-to-ground fault.
Figure 2 shows amplitude of 2nd zero-sequence current is higher those of 1st zero-sequence current for the difference between the capacitance to earth of 1st and 2rd. When single-phase grounding fault occurs, the varying the phase, amplitude and frequency of the zero-se- quence measurement current voltage of the non-fault feeder is not remarkable. However, The compensation current of the arc-suppressing coil makes the phase and amplitude of the zero-sequence measurement current of fault feeder to vary significantly. Experimental results verify the validity of zero sequence current increment method described in the previous sections.

\subsection{Dynamic Response of the Device}

In order to verify the Function of fault-line selection device based on integrative line selection strategy, the device is used to detect fault-feeder for one-phase-toground fault is used on the simulation of $1.2 \mathrm{kV}$ power network.

In this experimentation, the behavior of the device has been studied when single-phase grounding fault suddenly occur.

When a sudden one-phase-to ground fault happens, the zero-sequence current varies instantaneously. In this case, it can be observed how zero-sequence current only needs $30 \mathrm{~ms}$ to arrive to the step of the compensation of the arc-suppressing coil from transience. During the time, the difference between tamplitude of the transient current of with that of stable current for fault feeder is remarkable. Tamplitude of the transient current much higher than that of stable current for fault feeder. After MHK series controller of arc suppression coil achieved, the compensation current of the arc-suppressing, zero-sequence current is step of stable current in 5 cycle.

Figures $\mathbf{3}$ and $\mathbf{4}$ show the direction of zero-sequence transient current of fault feeder is opposite to that of the zero-sequence transient current of the non-fault feeder. Ideally, the result is coincident with the theoretical analysis

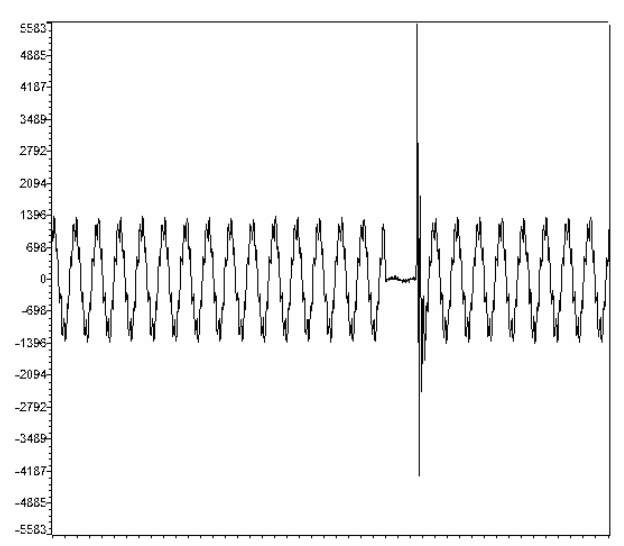

Figure 3. Zero voltage and current waves recording of nonfault-feeder. 


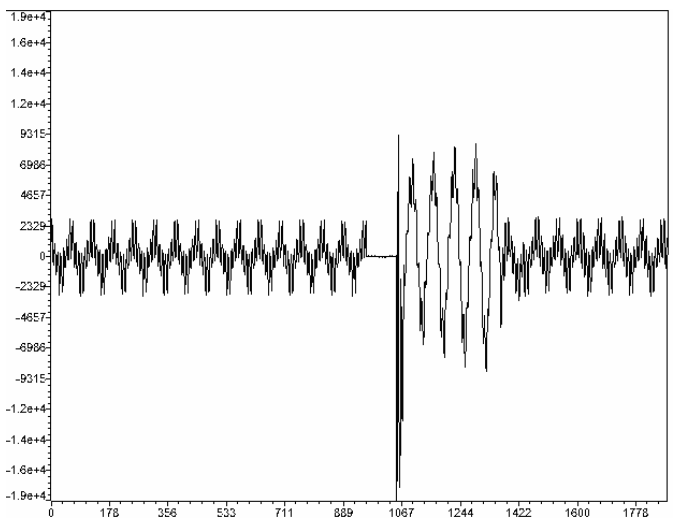

Figure 4. Zero voltage and current waves recording of faultfeeder.

of detecting earthed fault feeder the transient current direction.

Figure 4 shows experimental wareform of the zerosequence transient current of the fault feeder. As shown in this waveform, single-phase grounding fault results in more higher zero-sequence current increment. The fault duration was extended to $30 \mathrm{~ms}$ to allow the zero-sequence current to reach the new operating state. We compared the monitoring results from fault wareform recording with the experimental one. Consequently, two values were very matched.

\section{Conclusions}

In this paper, integrative line selection strategy, which is zero sequence current increment method and the direction of transient current, been proposed. Based on the integrative line selection strategy, the device of fault-line selection for one-phase-to ground fault on resonance grounding system is designed. The result obtained from the experiment carried out on the simulation of $1.2 \mathrm{kV}$ power network is as follows.
1) Experimental result of the selection device has been compared with real wave recording of fault wareform recording. As a result, two values were very matched. So, we confirmed the feasibility of the proposed fault-line selection device.

2) The integrative line selection strategy-zero sequence current increment method and the direction of transient current - been proposed. Real wareform of fault wareform recording confirm that the integrative line selection strategy is more stable and accurate method comparison with the single method.

\section{REFERENCES}

[1] H. Li, Y. Tang and C. Q. I.Sun, "Line Selection Device fo Small Current Single-phase Grounding Fault Bsed on Integrative Line Seletion Strategy"Industry and Mine Atomation, pp. 35-38, April 2012.

[2] X. C. Wang, “Zero Sequence Current Incremental Ratio Method in Small Current Grounding Fault Line Identification," Vol. 20. Power System Protecetion and control, October 2011, pp.125-129.

[3] H. C. Su, "Fault Line Seletion of Distribution Power System," China Machine press, Beijing 2008.

[4] K. Cheng and Y. Tang, "Single-Phase-to-Ground Fault Protection for Indirect Grounding Power System,” High Voltage Engineering.

[5] J. LEE, "The Research on Arc Suppression Coil and Line Selection Control Technology in Distribution Network," North China Electric Powe Unversity, 2007.

[6] Y. Yorozu, M. Hirano, K. Oka and Y. Tagawa, "Electron Spectroscopy Studies on Magneto-Optical Media and Plastic Substrate Interface,” IEEE Transl. Journal of Magnetics in Japan, Vol. 2, No. 8, 1987, pp. 740-741. doi:10.1109/TJMJ.1987.454959

[7] M. Young, “The Technical Writer's Handbook,” Mill Valley, CA: University Science, 1989. 